PROCEEDINGS OF THE

AMERICAN MATHEMATICAL SOCIETY

Volume 124, Number 10, October 1996

\title{
CHARACTERIZATION OF CLASSICAL GROUPS BY ORBIT SIZES ON THE NATURAL MODULE
}

\author{
MARTIN W. LIEBECK
}

(Communicated by Ronald M. Solomon)

\begin{abstract}
We show that if $V$ is a finite vector space, and $G$ is a subgroup of $P \Gamma L(V)$ having the same orbit sizes on 1-spaces as an orthogonal or unitary group on $V$, then, with a few exceptions, $G$ is itself an orthogonal or unitary group on $V$.
\end{abstract}

Let $C$ be a finite orthogonal or unitary group, with associated vector space $V$, and let $G$ be a subgroup of $P \Gamma L(V)$ having the same orbit sizes as $C$ on the set of 1-dimensional subspaces of $V$. We shall show that, with a few exceptions, under these hypotheses $G$ must itself be an orthogonal or unitary group on $V$. The precise result is stated in the Theorem below.

This paper was written in response to a question of Prof. S. Abhyankar, who makes use of the result in $[\mathrm{Ab}]$.

Theorem. Let $q$ be a prime power, $d \geq 3$ an integer, and $V=V_{d}(q)$ a vector space of dimension $d$ over $\mathbb{F}_{q}$. Suppose that $G$ is a subgroup of $P \Gamma L_{d}(q)$ such that the sizes of the orbits of $G$ on the 1-spaces of $V$ are as in one of cases (1) - (5) in Table 1 below. If $d \leq 7$, assume that $q>2$; and if $d \leq 4$, assume that $q>3$.

(a) If the orbit sizes are as in (4) or (5) of Table 1, then either $G \triangleright P S U_{d}\left(q^{1 / 2}\right)$, or $d=3, q=4, G \triangleright 3^{2}=O_{3}\left(P S U_{3}(2)\right)$ (and if also $G \leq P G L_{3}(4)$, then $G \triangleright$ $\left.\mathrm{PSU}_{3}(2)\right)$.

(b) If the sizes are as in (2), then either $G \triangleright P \Omega_{2 m}^{+}(q)$, or $G \triangleright P S U_{m}(q)$ (m even), or $d=8, G \triangleright \Omega_{7}(q)$ (embedded irreducibly in $P S L_{8}(q)$ ), or $d=4, q=$ 5, $G \triangleright A_{6}$.

(c) If the sizes are as in (3), then either $G \triangleright P \Omega_{2 m}^{-}(q)$, or $G \triangleright P S U_{m}(q)$ (m odd), or $d=4, G \triangleright{ }^{2} B_{2}(q)$ ( $q$ an odd power of 2 ), or $d=6, q=3, G \triangleright L_{3}(4)$, or $d=4, q=4, G \leq \Gamma L_{1}\left(2^{8}\right)$.

(d) If the orbit sizes are as in (1) with $q$ odd, then either $G \triangleright \Omega_{2 m+1}(q)$, or $d=7, G \triangleright G_{2}(q)$.

(e) If the orbit sizes are as in (1) with q even, then $G \triangleright S p_{2 a}\left(q^{b}\right)(2 a b=2 m)$ or $G \triangleright G_{2}\left(q^{b}\right) \quad(6 b=2 m)$, embedded naturally in $S p_{2 m}(q)$ acting indecomposably on $V$ (fixing a $2 m$-dimensional subspace $W$, with $V / W$ trivial).

Conversely, all the groups arising in (a)-(e) do have orbit sizes as in Table 1.

Remarks. 1. The classical groups arising in conclusions (a)-(d) all act in the natural way on $V$.

Received by the editors March 20, 1995.

1991 Mathematics Subject Classification. Primary 20G40.

(C)1996 American Mathematical Society 
2. If we relax the assumptions in the Theorem made on $q$ for small $d$, more examples occur, but it would not be hard to list these.

TABLE 1

\begin{tabular}{|c|c|c|}
\hline Case & $d$ & orbit sizes \\
\hline (1) & $2 m+1$ & $\frac{q^{2 m}-1}{q-1}, \frac{1}{2} q^{m}\left(q^{m}+1\right), \frac{1}{2} q^{m}\left(q^{m}-1\right)$ \\
\hline$(2)$ & $2 m$ & $\frac{\left(q^{m}-1\right)\left(q^{m-1}+1\right)}{q-1}, q^{m-1}\left(q^{m}-1\right)$ or \\
\hline$(3)$ & $2 m$ & $\begin{array}{l}\frac{\left(q^{m}+1\right)\left(q^{m-1}-1\right)}{q-1}, \frac{1}{2} q^{m-1}\left(q^{m}-1\right), \frac{1}{2} q^{m-1}\left(q^{m}-1\right)(q \text { odd }) \\
\frac{\left(q^{m}+1\right)\left(q^{m-1}-1\right)}{q-1}, \frac{1}{2} q^{m-1}\left(q^{m}+1\right) \text { or } \\
\left.\frac{1}{m}+1\right), \frac{1}{2} q^{m-1}\left(q^{m}+1\right)(q \text { odd })\end{array}$ \\
\hline$(4)$ & $2 m$ & $\frac{\left(q^{m}-1\right)\left(q^{m-\frac{1}{2}}+1\right)}{q-1}, \frac{q^{m-\frac{1}{2}}\left(q^{m}-1\right)}{q^{\frac{1}{2}}+1}(q$ square $)$ \\
\hline$(5)$ & $2 m+1$ & $\frac{\left(q^{m+\frac{1}{2}}+1\right)\left(q^{m}-1\right)}{q-1}, \frac{q^{m}\left(q^{m+\frac{1}{2}}+1\right)}{q^{\frac{1}{2}}+1}(q$ square $)$ \\
\hline
\end{tabular}

In the proof we shall use primitive prime divisors: if $n \geq 3$ and $(q, n) \neq(2,6)$, then by $[\mathrm{Zs}]$ there is a prime which divides $q^{n}-1$ but does not divide $q^{i}-1$ for $1 \leq i \leq n-1$. Such a prime is called a primitive prime divisor of $q^{n}-1$ and is denoted by $q_{n}$; note that $q_{n} \equiv 1 \bmod n$. Write $q_{n}^{*}$ for the product of all primitive prime divisors of $q^{n}-1$, counting multiplicities.

The proof of the Theorem is a fairly routine application of the results in [Li] and [GPPS]. The paper [Li] determines the irreducible subgroups of $P \Gamma L_{d}(q)$ having exactly two orbits on 1-spaces, and can be used to handle such cases in the Theorem. And [GPPS] lists subgroups of $P \Gamma L_{d}(q)$ which have order divisible by $q_{e}$ for some $e>\frac{1}{2} d$; since our group $G$ is in general divisible by such a prime, this applies to our problem. Despite the routine nature of the proof, we feel that the result may be of some interest, especially in view of the application [Ab].

Proof of the Theorem. Let $G \leq P \Gamma L_{d}(q)$ be as in the statement of the Theorem. Write $q=p^{f}$ with $p$ prime. Assume first that $d \geq 5$, and also that $q>2$ if $d \leq 8$; we shall handle the excluded cases later. Referring to Table 1 , we see that $|\bar{G}|$ is divisible by $q_{d}^{*}, q_{d-1}^{*}$ or $q_{d-2}^{*}$ in case $(1),(2)$ or $(3)$, and by $\left(q^{1 / 2}\right)_{2 d}^{*}$ or $\left(q^{1 / 2}\right)_{2 d-2}^{*}$ in case (4) or (5).

Suppose first that $G$ is reducible on $V$. Then $G \leq P_{i}$, the stabilizer in $P \Gamma L(V)$ of an $i$-space, for some $i$. The orbit sizes of $P_{i}$ on 1 -spaces are $\frac{q^{i}-1}{q-1}$ and $\frac{q^{i}\left(q^{d-i}-1\right)}{q-1}$, so one of these is an orbit size of $G$. The only possibility is that $d=2 m+1, i=2 m$ and the orbit sizes of $G$ are as in case (1) of Table 1 . Thus $G \leq P_{2 m}=Q L$, where $Q \cong\left(\mathbf{F}_{q}\right)^{2 m}$ is the unipotent radical and $L \triangleright S L_{2 m}(q)$ is a Levi subgroup. The orbit sizes of $P_{2 m}$ are $\frac{q^{2 m}-1}{q-1}, q^{2 m} ; G$ is transitive on the first orbit, and $Q$ is transitive on the second. Therefore $Q \not \leq G$. As $G$ acts irreducibly on $Q$, it follows that $G \cap Q=1$, whence $G$ is isomorphic to a subgroup of $L$ which is transitive on the 1-spaces of $V_{2 m}(q)$. The list of all transitive linear groups is given in [Li, Appendix 1], and the only possibilities which are divisible by the orbit sizes in (1), hence by $\frac{1}{2} q^{m}\left(q^{2 m}-1\right) /(q-1)$, are as follows:

(i) $G \leq \Gamma L_{1}\left(p^{2 f m}\right)\left(\right.$ where $\left.q=p^{f}\right)$; 
(ii) $G \triangleright S=S p_{2 a}\left(q^{b}\right)$ (where $\left.2 a b=2 m, a \geq 1\right)$;

(iii) $G \triangleright S=S L_{a}\left(q^{b}\right)$ (where $a b=2 m, a \geq 3$ );

(iv) $G \triangleright S=G_{2}\left(q^{b}\right)$ (where $6 b=2 m, q$ even).

In case (i) the divisibility condition forces $p^{f m}$ to divide $4 \mathrm{fm}$, whence $p=$ 2 , $f m=4$; but the subgroup $G \cap G L_{1}\left(2^{8}\right)$, being of odd order, fixes a 1-space of $V$, so $G$ has an orbit size dividing $\left|G: G \cap G L_{1}\left(2^{8}\right)\right|$, hence dividing 8, which is not the case.

In case (iii), or in case (ii) with $q$ odd, we have $H^{1}(S, Q)=0$ by [JP]. It follows that the subgroup $S$ of $G$ is conjugate to a subgroup of $L$, hence fixes a 1-space of $V$. But then $G$ has an orbit of size dividing $|G: S|$, which is not so.

In the remaining cases ((ii) with $q$ even, and (iv)), $H^{1}(S, Q)$ has dimension 1 by [JP]; by the argument of the previous paragraph, $S$ does not fix a 1-space, so $S$ is not conjugate to a subgroup of $L$. It follows that conclusion (e) of the Theorem holds. Here $S$ lies in a subgroup $S p_{2 m}(q)$ acting indecomposably on $V$, with orbit sizes as in (1) of Table 1 and point stabilizers $P_{1}$ (a parabolic), $O_{2 m}^{+}(q)$ and $O_{2 m}^{-}(q)$. The group $S$ is transitive on each of the orbits, since $S p_{2 m}(q)$ factorizes as $S . P_{1}=S . O_{2 m}^{+}(q)=S . O_{2 m}^{-}(q)$ (see [LPS, Tables 1 and 2]).

Now assume that $G$ is irreducible on $V$. Write $Z=Z\left(G L_{d}(q)\right)$. Choose an integer $b$, maximal such that $G \leq \Gamma L_{a}\left(q^{b}\right) / Z(a b=d)$ in its usual embedding in $P \Gamma L_{d}(q)$. If $a=1$ then $|G|$ divides $\left(q^{d}-1\right) d f$ (where $q=p^{f}$ ), which is impossible since orbit sizes in Table 1 divide $|G|$. Hence $a \geq 2$.

The subgroups of $\Gamma L(V)$ having two orbits on 1-spaces, and their orbit sizes, are listed in [Li, Appendix 2]. A glance at this list shows that the only such groups having orbit sizes as in Table 1 satisfy conclusion (a), (b) or (c) of the Theorem. Thus we may assume that $G$ has three orbits on 1-spaces; the orbit sizes are then as in (1), (2) or (3) of Table 1, with $q$ odd in cases (2), (3). In particular, $|G|$ is divisible by $q_{e}^{*}$, where $e=d, d-1$ or $d-2$.

Let $X$ be one of the classical groups $S L_{a}\left(q^{b}\right), S p_{a}\left(q^{b}\right), \Omega_{a}\left(q^{b}\right), U_{a}\left(q^{b / 2}\right)$, chosen to be minimal such that $G \leq N_{\Gamma L(V)}(X) / Z$. Write $\bar{X}=X / X \cap Z$. If $G$ contains $\bar{X}$ then $X$ must be orthogonal or unitary, and from the orbit sizes of $X$ we see that $b=1$ in the orthogonal case, $b=1$ or 2 in the unitary case; hence $G$ is as in (a)-(d) of the Theorem. Consequently we may assume that $\bar{X} \not \leq G$.

At this point we apply the main result of [As] on the subgroups of the classical group $N_{P \Gamma L(V)}(\bar{X})$. According to this result, either $G$ lies in a member of one of the families $\mathcal{C}_{1}, \ldots, \mathcal{C}_{8}$ of subgroups of this group, or $G \in \mathcal{S}$, a certain collection of almost simple subgroups. A discussion of this result can be found in [KL, Chapter 1], and detailed descriptions of the members of the families $\mathcal{C}_{i}$ in [KL, Chapter 4].

Suppose first that $G \in \mathcal{C}_{i}$ for some $i$. As $G$ is irreducible, and by choice of $b$ and $X, i$ is not 1,3 or 8; also subgroups in $\mathcal{C}_{i}$ for $i=4,5,7$ do not have order divisible by $q_{e}^{*}$. If $G \leq M \in \mathcal{C}_{2}$, then $G$ stabilizes a decomposition $V=V_{1} \oplus \ldots \oplus V_{k}$, where each $V_{i}$ has $\mathbb{F}_{q}$-dimension $r, r k=d$ and $G \cap P G L(V) \leq\left(G L_{r}(q)\right.$ wr $\left.S_{k}\right) / Z$. As $q_{e}^{*}$ divides $|G|$ and $e \geq d-2>d / 2$, we must have $r=1, k=d$ and $q_{e}^{*}=d-1$ or $d$. But $G$ has at least $k$ orbits on 1 -spaces, so this is impossible when $d \geq 5$. Finally, suppose that $G \leq M \in \mathcal{C}_{6}$. Then $|M \cap P G L(V)|$ divides $r^{2 k}\left|S p_{2 k}(r)\right|$, where $r$ is prime, $a=r^{k}$ and $r \mid q^{b}-1$. Since $q_{e}^{*}$ divides $|G|$, this means that $r=2, a=d=2^{k}$ and $q_{e}^{*}=2^{k} \pm 1$ with $e=d$ or $d-2$. A result of Hering [He, 3.9] determines all $(q, e)$ such that $q_{e}^{*}=e+1$, and this implies that $(q, e)=(3,4),(3,6)$ or $(5,6)$. In the first case $d=4$, contrary to assumption; in the second case $G \leq 2^{6} . S p_{6}(2)<L_{8}(3)$, and 
the orbit sizes of $2^{6} . S p_{6}(2)$ on 1-spaces are 720,2560 by [Li, Appendix 2], neither of which is an orbit size of $G$; and in the last case the orbit sizes of $G$ do not divide $2^{6}\left|S p_{6}(2)\right|$.

It remains to deal with the case where $G \in \mathcal{S}$. Here $G$ is almost simple; write $S=F^{*}(G)$. In [GPPS, Examples 2.6 - 2.9], all possibilities for subgroups in $\mathcal{S}$ which are divisible by a primitive prime divisor $q_{i b}, i>a / 2$, are listed. Clearly $e=i b$ with $i>a / 2$, so our group $S$ is in this list.

Suppose first that $S$ is of Lie type in characteristic $p$. Then $S$ is given by [GPPS, Example 2.8]. The only possibilities with $|S|$ divisible by $q_{e}^{*}(e \geq d-2)$ are $(S, d, e)=$ $\left(L_{2}\left(q^{3}\right), 8,6\right),\left(\Omega_{7}(q), 8,6\right),\left(G_{2}(q)\right.$ or $\left.{ }^{2} G_{2}(q), 7,6\right)\left(q\right.$ odd), $\left(G_{2}\left(q^{2}\right), 14,12\right)$ ( $q$ odd), $\left(U_{3}(q), 8-\delta_{3, p}, 6\right)$ or $\left(U_{3}\left(q^{2}\right), 14,12\right)(p=3)$. Of these, the only cases where $\mid$ Aut $S \mid$ is divisible by the orbit sizes in Table 1 are $S=\Omega_{7}(q), G_{2}(q)$, as in conclusions (b), (d) of the Theorem. In these cases $S$ lies in $\Omega_{8}^{+}(q), \Omega_{7}(q)$ respectively, and is transitive on each orbit of these groups on 1-spaces (see [LPS] again).

Now assume that $S$ is alternating, sporadic, or of Lie type in $p^{\prime}$-characteristic. From the lists in [GPPS, Examples 2.6, 2.7 and 2.9], we see that one of the following holds:

(i) there is only one primitive prime divisor of $q^{e}-1$ dividing $|G|$, and this is equal to $e+1$ or $2 e+1$; moreover, this prime divides $|G|$ to the first power only;

(ii) $S=L_{2}(s)$ with $s$ prime, $d=(s \pm 1) / 2, e=(s-1) / 2$ and $q_{e}^{*}=(e+1)(2 e+1)$.

Consequently $q_{e}^{*}$ must be $e+1,2 e+1$ or $(e+1)(2 e+1)$. Hence the possibilities for $(q, e)$ are given by $[\mathrm{He}, 3.9]$. In case $(\mathrm{ii}),(q, e)$ is $(3,18)$ or $(17,6)$. But then either $S=L_{2}(37)<L_{d}(3)(d=18$ or 19$)$, or $S=L_{2}(13)<L_{d}(17)(d=6$ or 7$)$, and $\mid$ Aut $S \mid$ is not divisible by the orbit sizes. Therefore (i) holds, and the possibilities for $q, e$ are as follows:

$$
\begin{array}{ll}
q=2: & e=3,4,8,10,12,18 \text { or } 20 \\
q=3: & e=4 \text { or } 6 \\
q=4: & e=3 \text { or } 6 \\
q=5: & e=6 .
\end{array}
$$

Suppose that $q=2$ or 4 . Since we are assuming $G$ to have three orbits on 1 -spaces, we must have $d=2 m+1$ and $|G|$ divisible by the orbit sizes $\frac{q^{2 m}-1}{q-1}, \frac{1}{2} q^{m}\left(q^{m}+1\right)$, $\frac{1}{2} q^{m}\left(q^{m}-1\right)$. (In particular, $e=2 m=d-1$.) From [GPPS], we see that the only possibilities for $S=F^{*}(G)$ satisfying these conditions are $S=P S p_{4}(5)$ or $P S p_{6}(3)$, with $q=2, d=13$. However, $P S p_{4}(5), P S p_{6}(3)$ are not subgroups of $L_{13}(2)$ by Lagrange's theorem.

Now let $q=3$. Here $e=4$ or 6 , so $5 \leq d \leq 8$. If $d=5$, the orbit sizes $40,45,36$ divide $|G|$, and [GPPS] shows that $S=M_{11}$; however, $M_{11}<L_{5}(3)$ has only two orbits on 1-spaces, by [Li]. If $d=6$ then the orbit sizes of $G$ are $130,117,117$ or 112 , 126,126 , whence $|G|$ is divisible by either $3^{2} .5 .13$ or $2^{4} .3^{2} .7$; now [GPPS] implies that $S$ is $L_{3}(4), A_{7}$ or $J_{2}$. The first case is in conclusion (c) of the Theorem, and $L_{3}(4)<\Omega_{6}^{-}(3)$ is transitive on each $\Omega_{6}^{-}(3)$-orbit on 1 -spaces (see [At, p. 52]). In the second case, $G=A_{7}$ or $S_{7}$ has orbit sizes $112,126,126$; but $A_{7}$ and $S_{7}$ have no transitive actions of degree 112 (see [At, p.10]). Finally, $J_{2} \not \leq L_{6}(3)$ by Lagrange's theorem. When $d=7$, [GPPS] gives no possibilities for $G$ of order divisible by the orbit sizes $2^{2} .7 .13,2.3^{3} .7,3^{3} .13$. Now suppose $d=8$. The orbit sizes imply that $|G|$ is divisible by either $2^{5} .3^{3} .5 .7$ (case (2) of Table 1 ) or by 13.41 (case (3)). By [GPPS], the former holds, and $S=S p_{6}(2), \Omega_{8}^{+}(2), A_{9}$ or $L_{3}(4)$. In the first three cases the 8dimensional representation of $S$ is uniquely determined (see the 3 -modular character 
tables of these groups in [At2]), and embeds $S \leq \Omega_{8}^{+}(2)<\Omega_{8}^{+}(3)<L_{8}(3)$. But in this representation, $\Omega_{8}^{+}(2)$ has an orbit of size 120 on 1-spaces (see [Li, p.505, case $3(\mathrm{e}, \mathrm{f})]$ ). Finally, if $S=L_{3}(4)$, one checks using [At, p.23] that the group $G$ with $F^{*}(G)=S$ has no transitive action of degree $2^{3} .3^{3} .5$, which is one of the required orbit sizes.

Now suppose that $q=5$. Then $e=6$ and $6 \leq d \leq 8$. When $d=6,|G|$ is divisible by either 13.31 (case (2) of Table 1) or $2^{2} .3^{3} .5^{2} .7$ (case (3)). By [GPPS], the latter holds, and $S=J_{2}$. But $J_{2}<L_{6}(5)$ has only two orbits on 1-spaces, by [Li]. Finally, when $d=7$ or 8 , [GPPS] shows that there are no possibilities for $S$ with $|G|$ divisible by the required orbit sizes.

This completes the proof of the Theorem under our initial assumption that $d \geq 5$ and that $q>2$ if $d \leq 8$. By the hypotheses of the Theorem, it remains to handle the cases $d=3,4$ with $q \geq 4$, and $d=8, q=2$. The argument given at the beginning of the proof of the Theorem (second paragraph) shows that if $G$ is reducible then $d=3, q$ is even and $G \triangleright S p_{2}(q)$ as in conclusion (e). Thus we suppose that $G$ is irreducible. In the case $d=8, q=2, G$ has two orbits on 1-spaces, and we check that conclusion (b) or (c) holds using [Li]. Thus we suppose from now on that $d=3$ or 4 and $q \geq 4$.

As in the proof above, we choose $b$ maximal such that $G \leq \Gamma L_{a}\left(q^{b}\right) / Z$, where $a b=d$. If $a=1$ then the orbit sizes must divide $\left(q^{d}-1\right) \cdot d \log _{p} q$, which implies that $d=4$ and $q=4,8$ or 16 (and the orbit sizes are as in (3) of Table 1). The subgroups of $\Gamma L_{1}\left(q^{d}\right)$ having two orbits on nonzero vectors are given by [FK, §3], from which we see that an example arises if and only if $q=4$, as in conclusion (c).

Hence we now assume that $a \geq 2$; and $(a, b)=(d, 1)$ or $(2,2)$. Again choose a classical group $X$ of dimension $a$ over $\mathbf{F}_{q^{b}}$, minimal such that $G \leq N_{\Gamma L(V)}(X) / Z$. If $G$ contains $\bar{X}=X / X \cap Z$, then one of (a)-(d) of the Theorem holds, so assume $\bar{X} \not \subset G$. Suppose that $G$ is contained in a member $M$ of one of the families $\mathcal{C}_{i}$ of subgroups of $N(\bar{X})$. Then $i \neq 1,3,4,7,8$ by choice of $b$ and $X$. If $i=2$ or 5 then the orbit sizes of $M$ are not compatible with those of $G$. And if $M \in \mathcal{C}_{6}$, then $\left|G \cap L_{d}(q)\right|$ divides $2^{4} .3^{3}$ (if $d=3$ ), or $2^{8} .3^{2} .5$ (if $d=4$ ). The fact that $|G|$ is divisible by orbit sizes in Table 1 forces either $d=3, q=4$ or $d=4, q=5$. In the first case, $G \leq 3^{2} .2 S_{4}$ (see [At, p.23]) and $G$ has orbit sizes 9,12 as in (5) of Table 1. Then clearly $G \triangleright 3^{2}$, as in (a) of the Theorem; moreover, if also $G \leq P G L_{3}(4)$, then $G \leq 3^{2} .2 A_{4}$, whence from the action on the orbits, $G$ contains $3^{2} \cdot Q_{8}=P S U_{3}(2)$. Now consider $d=4, q=5$. Here $G \leq 2^{4} \cdot S p_{4}(2)$ and the orbit sizes of $G$ are $36,60,60$ or 36,120 . By [Li], the group $2^{4} . S p_{4}(2)$ has orbit sizes 60,96 . The normal subgroup $2^{4}$ has 15 orbits of size 4 and 16 of size 6 , both sets permuted transitively by the factor $S p_{4}(2)$ (see [Li, 1.2]). Hence $G$ cannot contain this $2^{4}$, and so $G \cap 2^{4}=1$. Thus $G=A_{6}$ or $S_{6}$, as in conclusion (b) of the Theorem.

Thus $G$ lies in the collection $\mathcal{S}$ of almost simple subgroups of $N_{P \Gamma L(V)}(\bar{X})$. Let $S=F^{*}(G)$. For $d \leq 4$, the members of this collection are well known, and are among the following (see [Kl, Chapter 5$]$ ):

$$
\begin{array}{ll}
d=3: & S=A_{5}, A_{6} \text { or } L_{2}(7) \\
d=4: & S=A_{5}, A_{6}, A_{7}, L_{2}(7), U_{4}(2), L_{3}(4) \text { or } L_{2}(q) .
\end{array}
$$

By [Li], the only possibility for $G$ having two orbits is $d=4, q=5, S=A_{6}$, as in (b) of the Theorem. So assume that $G$ has three orbits; these have sizes $q+1$, $\frac{1}{2} q(q+1), \frac{1}{2} q(q-1)$, or $(q+1)^{2}, \frac{1}{2} q\left(q^{2}-1\right), \frac{1}{2} q\left(q^{2}-1\right)$, or $q^{2}+1, \frac{1}{2} q\left(q^{2}+1\right)$, $\frac{1}{2} q\left(q^{2}+1\right)$. The only possibilities with $\mid$ Aut $S \mid$ divisible by orbit sizes are as 
follows:

$$
\begin{aligned}
& d=3, q=4, S=A_{5} \text { or } A_{6}, \text { and } \\
& d=4, q=5, S=A_{6} \text { or } A_{7} .
\end{aligned}
$$

In the case $d=3, A_{6}$ has orbit sizes 6,15 (see [Li]), and there is no irreducible $A_{5}$ in $L_{3}(4)$. And in the case $d=4, A_{6}$ has two orbits $([\mathrm{Li}])$ and there is no $A_{7}$ in $L_{4}(5)$.

This completes the proof of the Theorem.

\section{REFERENCES}

[Ab] S. Abhyankar, "Again nice equations for nice groups", Proc. Amer. Math. Soc. 124 (1996), 3567-3576.

[As] M. Aschbacher, "On the maximal subgroups of the finite classical groups", Invent. Math. 76 (1984), 469-514. MR 86a:20054

[At] J.H. Conway, R.T. Curtis, S.P. Norton, R.A. Parker and R.A. Wilson, Atlas of finite groups, Oxford University Press, 1985. MR 88g:20025

[FK] D.A. Foulser and M.J. Kallaher, "Solvable, flag-transitive, rank 3 collineation groups", Geom. Ded. 7 (1978), 111-130. MR 57:12263

[GPPS] R. Guralnick, T. Penttila, C.E. Praeger and J. Saxl, "Linear groups with orders having certain primitive prime divisors", preprint, University of Western Australia, 1994.

[He] C. Hering, "Transitive linear groups and linear groups which contain irreducible subgroups of prime order", Geom. Ded. 2 (1974), 425-460. MR 49:439

[At2] C. Jansen, K. Lux, R.A. Parker and R.A. Wilson, Atlas of modular characters, Oxford University Press, 1995.

[JP] W. Jones and B. Parshall, "On the 1-cohomology of finite groups of Lie type", Proc. Conf. on Finite Groups, eds. W. Scott and F. Gross, Academic Press, (1976). MR 53:8272

[Kl] P.B. Kleidman, The subgroup structure of some finite simple groups, Ph.D. Thesis, University of Cambridge, 1987.

[KL] P.B. Kleidman and M.W. Liebeck, The Subgroup Structure of the Finite Classical Groups, London Math. Soc. Lecture Note Series 129, Cambridge University Press, Cambridge, 1990. MR 91g:20001

[Li] M.W. Liebeck, "The affine permutation groups of rank three", Proc. London Math. Soc. 54 (1987), 477-516. MR 88m:20004

[LPS] M.W. Liebeck, C.E. Praeger and J. Saxl, "The maximal factorizations of the finite simple groups and their automorphism groups", Mem. Amer. Math. Soc. 86, No. 432 (1990), 1-151. MR 90k:20048

[Zs] K. Zsigmondy, "Zur Theorie der Potenzreste", Monatsh. fur Math. und Phys. 3 (1892), 265-284.

Department of Mathematics, Imperial College, London SW7 2BZ, United Kingdom

E-mail address: m.liebeck@ic.ac.uk 